\title{
PABLO MONTESINO Y LA FORMACIÓN MATEMÁTICA DEL MAGISTERIO EN ESPAÑA (1838-1850)
}

\author{
PABLO MONTESINO E A FORMAÇÃO MATEMÁTICA DE PROFESSORES EM \\ ESPANHA (1838-1550)
}
PABLO MONTESINO AND THE MATHEMATICAL TRAINING OF PRIMARY SCHOOL TEACHERS IN SPAIN (1838-1850)

Dolores Carrillo Gallego *

José Ginés Espín Buendía *

\begin{abstract}
RESUMEN
Las Escuelas Normales fueron creadas en España por el Plan de Instrucción Primaria de 1838 como instituciones de formación de maestros. Sus profesores se formaron en la Escuela Normal Central que comenzó a funcionar en 1839 en Madrid. Las Escuelas Normales proporcionaron, por primera vez, conocimientos profesionales a los maestros; en particular, saberes profesionales como docentes de matemáticas en las escuelas primarias. Este artículo analiza cuál fue la influencia de Pablo Montesino en la configuración de los «saberes para enseñar» matemáticas que se consideraron necesarios para un maestro de escuela primaria. Pablo Montesino fue el primer Director de la Escuela Normal Central y el experto que influyó en la orientación y desarrollo de la educación primaria y la formación de los maestros a través de su participación en comisiones y asociaciones, de su docencia en la Escuela Normal Central, mediante la publicación de libros, artículos e, incluso, de documentos legislativos.
\end{abstract}

Palabras clave: Experto. Saber para enseñar. Formación de maestros. Escuelas Normales.

\section{RESUMO}

As Escuelas Normales foram criadas na Espanha pelo Plan de Instrucción Primaria de 1838, como instituições de formação de professores. Os seus professores foram formados na Escuela Normal Central que começou a funcionar em 1839 em Madrid. As Escuelas Normales forneceram, pela primeira vez, conhecimentos profissionais aos professores; em particular, conhecimentos profissionais como professores de matemática nas escolas primárias. Este artigo analisa a influência de Pablo Montesino na formação do "conhecimento para ensinar" matemática, que era considerado necessário para um professor de escola primária. Pablo Montesino foi o primeiro Diretor da Escuela Normal Central, e o especialista que influenciou a orientação e o desenvolvimento do ensino primário, da formação de professores através da sua participação em comissões e associações, do seu ensino na Escola Normal Central, mediante a a publicação de livros, artigos e até documentos legislativos.

Palavras-chave: Perito. Saber ensinar. Formação de professores. Escolas de Formação de Professores.

\footnotetext{
* Doctora en Pedagogía (Universidad de Murcia). Profesora Titular de Didáctica de las Matemáticas. Facultad de Educación (Universidad de Murcia). Campus Universitario de Espinardo, 30100, Murcia (España). Correo: carrillo@um.es

** Doctor en Matemáticas (Universidad de Murcia). Profesor de Educación Secundaria. IES Los Molinos (Consejería de Educación y Cultura de la Región de Murcia). C/Paz de Aquisgran s/n, Cartagena (España). Correo: josegines.espin@murciaeduca.es
}

Universidade Federal de Mato Grosso, Cuiabá, Mato Grosso, Brasil. Revista REAMEC - Rede Amazônica de Educação em Ciências e Matemática, v. 9, n. 3, e21087, setembro-dezembro, 2021. 


\section{ABSTRACT}

The Escuelas Normales were created in Spain by the Plan de Instrucción Primaria in 1938 as institutions for the training of teachers. Their teachers graduated from the Escuela Normal Central that began to work in 1839 in Madrid. The Escuelas Normales provided, for the first time, professional knowledge to teachers; in particular, professional knowledge as mathematics teachers in primary school. This paper analyses how Pablo Montesino influenced in the configuration of «knowledge for teaching" mathematics which was considered necessary for a primary school teacher. Pablo Montesino was the first Head Teacher of the Escuela Normal Central and the expert who influenced the orientation and development of primary education and the training of teachers through his participation in commissions and associations, his teaching at the Escuela Normal Central, the publication of books, papers and even legislative documents.

Keywords: Expert. Knowledge for theaching. Teaching training. Normal Schools.

\section{CONSIDERACIONES INICIALES}

El Plan de Instrucción Primaria de 21 de julio de 1838 (Colección, 1856, p. 3-11), al crear las Escuelas Normales (EENN) para la formación de los maestros de enseñanza primaria en España, supuso un cambio muy significativo en la formación y el reclutamiento de estos docentes, pues anteriormente los maestros obtenían su acreditación simplemente con un examen sobre contenidos de las escuelas de primeras letras (CARRILLO, 2005, p. 11-30) que, generalmente, se reducía a escribir al dictado y realizar algunas operaciones aritméticas. En las EENN se implementaron los primeros planes de estudio enfocados a la formación profesional de los maestros; estos planes fueron el punto de partida y el modelo de los que se han formulado, a lo largo de los años, para la formación del magisterio. Ese mismo Plan de 1838 creó una Escuela Normal Central (ENC) para la formación del profesorado de las EENN provinciales; el primer director de esta institución fue Pablo Montesino, persona que formó parte de diversas comisiones relacionadas con la educación y a quien se atribuye la redacción de varios documentos ministeriales; seguramente fue el principal experto al que se recurrió desde el Gobierno para las cuestiones relativas a la enseñanza primaria.

El papel de los expertos en la configuración de los saberes, en particular de los saberes profesionales de los profesores que enseñan matemáticas está siendo objeto de investigación por parte de personas ligadas al GHEMAT (ALMEIDA y VALENTE., 2019; MORAIS y VALENTE, 2020) los cuales destacan el interés de estudiar la acción e influencia de los expertos que influyeron en la configuración de los planes de estudio y demás documentos curriculares y su influencia en el proceso de institucionalización de la formación del profesorado en Brasil. Burke (2017) relaciona los términos expert y expertise con una práctica 
que surgió en Inglaterra en el siglo XIX, «la creciente utilización, por parte de los gobiernos, de asesoramiento especializado para encarar problemas prácticos» (p. 59) y considera esas prácticas como un inicio de la profesionalización de un saber determinado; esta situación que comenta Burke nos proponemos estudiarla en España, a través del experto Pablo Montesino y el inicio de la profesionalización de la Pedagogía en el marco de las instituciones de formación del profesorado primario.

El objetivo de este trabajo es estudiar la influencia de un experto, Pablo Montesino, en la organización de las Escuelas Normales así como sus propuestas sobre los saberes profesionales en la formación de maestros como docentes de matemáticas en la educación primaria. Las principales fuentes del trabajo son documentos elaborados por Montesino: libros, como el Manual para los maestros de las escuelas de párvulos (MONTESINO, 1850 y 1992), los artículos que publicó en el Boletín Oficial de Instrucción Pública (MONTESINO, 1843) y, sobre todo, sus apuntes de la asignatura de pedagogía, editados por Anastasio Martínez Navarro (MONTESINO, 1988).

Para analizar las propuestas de Montesino sobre la formación de los maestros como docentes de matemáticas en la educación primaria se ha utilizado una diferenciación entre el saber $a$ enseñar y el saber para enseñar. Shulman (1987) estudió los tipos de conocimiento que se utilizan en el ejercicio de la docencia, diferenciando siete categorías de conocimientos, entre ellas, lo que denominó el conocimiento pedagógico del contenido (Pedagogical Content Knowlwdge-PCK). Hofstetter y Schneuwly (2007), han diferenciado entre el saber $a$ enseñar (el saber que es el objeto de su trabajo) y el saber para enseñar (el saber que es la herramienta de su trabajo) y han estudiado los procesos de transformación de estos saberes y su implementación en la formación de los docentes.

En el caso de la enseñanza de las matemáticas, Ball, Thames y Phelps (2008) hablan del conocimiento matemático necesario para la enseñanza de las matemáticas (Mathematical Knowledge for Teaching, MKT), y diferencian cuatro dominios dentro del MKT: el conocimiento común del contenido (common content knowledge, CCK); el conocimiento especializado del contenido (specialized content knowledge, SCK); el conocimiento del contenido y de los estudiantes (knowledge of content and students, KCS); y el conocimiento del contenido y la enseñanza (knowledge of content and teaching, KCT). Por otro lado, Ciradde (2006), en su tesis doctoral, diferenció entre Matemáticas a enseñar, es decir las matemáticas que figuran en el currículum oficial; Matemáticas para el enseñante, que son las que un profesor debe conocer para poder comprender los contenidos del programa de estudios; y 
Matemáticas para la enseñanza, las que construye el profesor cuando se cuestiona las razones de ser de un conocimiento del currículo escolar.

Partiendo de los trabajos de Hofstetter y Schneuwly (2007, p. 198), Valente (2018) comenta el proceso de constitución de los saberes matemáticos profesionales, la matemática $a$ enseñar y la matemática para enseñar, y sostiene que los saberes profesionales son una construcción histórica en el seno de la profesión. Por ello señala la importancia de conocer los procesos mediante los cuales se han construido los saberes específicos de la profesión de enseñante de matemáticas, es decir, de la matemática a enseñar y de la matemática para enseñar, especialmente la relación entre ambos tipos de conocimiento en las instituciones dedicadas a la formación de profesores que enseñan matemáticas.

En el trabajo se han utilizado las categorías de saber para enseñar propuestas en Carrillo (2020), y que se basan en Baumert y Kunert (2016, citado en HOFSTETTER y SCHNEUWLY, 2007). Consideraremos, por tanto:

1) saber a enseñar: en nuestro caso, las matemáticas que son objeto de enseñanza en la escuela primaria; están fijadas por las leyes, los reglamentos o los planes de estudio, pero también por los libros de texto de la enseñanza primaria y otros documentos más o menos oficiales.

2) saber para enseñar: los saberes que, como consideran Hofstetter y Schneuwly (2007, p. 18), son «las herramientas de trabajo» de los docentes. Se han diferenciado tres tipos:

2a) saberes de las ciencias disciplinares: matemáticas para enseñar; se trata de profundizar en los contenidos de la enseñanza primaria, conocer las razones de ser de esos contenidos, técnicas alternativas a utilizar así como su justificación;

2b) saberes de las didácticas disciplinares: metodología de las matemáticas;

2c) saberes pedagógicos: teoría e historia de la educación; organización escolar; psicología infantil; psicología del aprendizaje; prácticas de enseñanza.

\section{PABLO MONTESINO, UN LIBERAL EXPERTO EN EDUCACIÓN}

Pablo Montesino (1781-1849) fue una persona clave para la enseñanza primaria y la formación de los maestros tras la muerte del rey de España, Fernando VII, en $1833^{1}$. Médico

\footnotetext{
${ }^{1}$ La vida y obra de Pablo Montesino ha sido objeto de varios trabajos como los que se presentaron en unas jornadas sobre él (VEGA, 1998) y los estudios introductorios de Anastasio Martínez Navarro (MARTÍNEZ NAVARRO, 
de profesión, fue Diputado durante el Trienio Liberal (1820-1822); al volver el régimen absolutista, en 1822 tuvo que exiliarse en Inglaterra donde conoció diversas experiencias educativas, en particular, las escuelas de enseñanza mutua y las escuelas de párvulos de Wilderspin. A su vuelta a España se involucró en actividades relacionadas con la educación, y, como afirma Escolano, está asociado a «la mayor parte de las innovaciones pedagógicas que acompañaron el despegue de nuestro sistema escolar primario en la primera etapa de la llamada 'era isabelina', es decir, en la fase progresista del nuevo régimen liberal impulsado por la burguesía revolucionaria» (ESCOLANO, 1998, p. 15).

En la primera mitad del siglo XIX, en España, los periodos de gobierno liberal se pueden denominar también constitucionales; en ellos, el poder real no era absoluto, pues existía una constitución (la de Cádiz, de 1812, la de 1837 o la de 1845) que fijaba sus límites. En todas las constituciones había apartados dedicados a la educación y correspondía al Estado promulgar las leyes que definieran un sistema educativo que diferenciara niveles educativos y los organizara de forma articulada. En esta nueva situación, los Gobiernos requirieron el asesoramiento de expertos que intervinieron en la redacción de los documentos legislativos. Uno de esos expertos fue Montesino.

Pablo Montesino influyó en el sistema educativo a través de varias vías; desde 1834 formó parte de la Comisión, asesora del Gobierno, encargada de redactar un plan de instrucción primaria siendo, según Antonio Gil de Zárate (1855, p. 256), el autor de la mayoría de los trabajos de la Comisión; en particular, en 1838 escribió el Reglamento de las escuelas públicas de instrucción primaria elemental de 26 de noviembre de 1838 (MINISTERIO, 1979, pp. 155190). Entre 1841 y 1845 dirigió el Boletín Oficial de Instrucción Pública (BOIP) donde publicó numerosos trabajos, entre ellos algunos sobre la enseñanza de las matemáticas en la educación primaria y en párvulos, así como otros sobre la organización de las EENN y la formación de los maestros. También tuvo una labor destacada en la Sociedad encargada de propagar y mejorar la educación del pueblo, que pretendía difundir en España las escuelas de párvulos y para la cual escribió un Manual para los maestros de las escuelas de párvulos (MONTESINO, 1850, 1992). Se interesó también por la enseñanza en la educación secundaria y la Universidad (MONTESINO, 1836). Desde 1839 fue Director de la ENC y redactor de sus Reglamentos de 1837 y 1842 (GACETA, 1837; REGLAMENTO, 1842), participando, asimismo en la

1988) a su Curso de Educación y el de Julio Ruiz Berrio (1992) a la reedición del Manual para los maestros de las escuelas de párvulos (MONTESINO, 1992). 
redacción del Reglamento orgánico para las Escuelas Normales de instrucción primaria de 13 de octubre de 1843 (Colección, 1856, p. 60-72) y del Reglamento para el régimen de las Escuelas Normales superiores y elementales de instrucción primaria de 15 de marzo de 1849 (Colección, 1856, pp. 95-102).

\section{LAS PRIMERAS ESCUELAS NORMALES EN ESPAÑA Y SUS ESTUDIOS}

El Plan de Instrucción Primaria de 1838 ordenaba una reestructuración profunda de la educación primaria en España. Para que esta propuesta legislativa tuviera posibilidades de éxito se exigía un cuerpo de maestros con una formación adecuada.

Con el fin de adecuar la formación de los maestros, el 16 de noviembre de 1838 se publicó un Reglamento de instrucción primaria (MINISTERIO, 1979, p. 155-190) cuya redacción se debe a Pablo Montesino. El Reglamento se refiere, exclusivamente, a las escuelas primarias elementales, que son las que «se establecen para la masa general del pueblo, y tienen por objeto desarrollar las facultades mentales del hombre, suministrando los conocimientos necesarios para todas las clases sin distinción» (MINISTERIO, 1979, p. 157). En el Reglamento se detallaba la organización de la escuela, el material necesario, el empleo del tiempo, la enseñanza de cada una de las materias, etc., pues el redactor consideraba «preciso no solo expresar las cosas que deben hacerse, sino la manera de hacerlas y la razón en que se fundan, por mas obvias que parezcan» (MINISTERIO, 1979, p. 155).

En el Preámbulo del Reglamento, Montesino justifica la necesidad de este documento por «establecer algunas prácticas poco conocidas por el mayor número de los maestros que han de adoptarlas» (MINISTERIO, 1979, p. 155); pues la mayor parte de los maestros estaban «faltos de la conveniente instrucción y de medios de adquirirla» (MINISTERIO, 1979, p. 155) . Es en este contexto, y ante esta necesidad, cuando se crean las EENN.

El 8 de marzo de 1839, bajo la dirección de Pablo Montesino, se inaugura la ENC en Madrid. La finalidad de esta primera Escuela Normal era la de formar profesores que nutrieran las futuras EENN distribuidas por el resto del país. En 1841 se gradúa la primera promoción de maestros de la ENC. De entre 67 aspirantes, obtuvieron el título de maestro un total de 43 estudiantes (que correspondían a 33 provincias, diez de ellas con dos alumnos). Con esta primera promoción de maestros se comienzan a crear el resto de EENN. En 1845, 42 EENN estaban ya en funcionamiento (ESCOLANO, 1982, p. 60). 
Con las EENN se pretendía mejorar la preparación de los maestros tanto en lo referente a aspectos curriculares como metodológicos; para ello, los planes de estudio incluían como materias las de la enseñanza primaria y una específica, la Pedagogía, dentro de la cual se estudiaban las metodologías de las diferentes materias escolares, entre ellas, las Matemáticas. En las siguientes dos listas se recogen las materias que se debían impartir en la ENC y en las EENN. MATERIAS DE ENSEÑANZA EN LA ESCUELA NORMAL CENTRAL, SEGÚN
LOS REGLAMENTOS DE 1837 Y 1842

Art. 22. Las materias de enseñanza indispensables son las siguientes:

1a . Religión y moral.

2a . Lengua castellana.

3a. Aritmética y elementos de geometría.

4a. Dibujo lineal.

5a. Elementos de física.

6a. Elementos de historia natural.

7a. Geografía é historia.

8a . Principios generales de educación moral, intelectual y física, con instrucciones especiales acerca de los medios más conducentes para conservar la salud de los niños y robustecerlos; o sea el modo de combinar los ejercicios gimnásticos ó corporales con juegos y ocupaciones ordinarias de la niñez.

9a. Métodos de enseñanza y pedagogía.

10a. Lectura.

11a. Escritura.

Art. 23. Podrá haber otras enseñanzas adicionales, particularmente de agrimensura y lengua francesa e inglesa, o la simple traducción de éstas, si la Junta de Estudios considera que pueden tener lugar; sin perjuicio de las de Reglamento, y si los fondos del Establecimiento lo permitiesen.

\section{MATERIAS DE ENSEÑANZA EN EL REGLAMENTO DE 1843}

Art. 5..$^{\circ}$ La enseñanza de las escuelas normales, para ser completa, ha de abrazar las materias siguientes:

$1 .^{\circ}$ Moral y religión.

$2 .^{\circ}$ Lectura y escritura.

$3 .^{\circ}$ Gramática castellana.

4. ${ }^{\circ}$ Leves nociones de retórica, poética y literatura española.

5. ${ }^{\circ}$ Aritmética y sus aplicaciones, con un conocimiento general de las principales monedas, pesos y medidas que se usan en las diferentes provincias de España.

6. ${ }^{\circ}$ Principios de geometría con sus aplicaciones a los usos comunes de la vida y de las artes industriales.

7. ${ }^{\circ}$ Dibujo lineal.

8. Aquellas nociones de física, química é historia natural indispensables para tener un conocimiento general de los fenómenos del universo, o hacer aplicaciones a los usos mas comunes de la vida.

9. ${ }^{\circ}$ Elementos de geografía e historia, sobre todo las de España.

$10 .^{\circ}$ Principios generales de educación y métodos de enseñanza, con su práctica en la escuelas de niños para los aspirantes a maestros.

Art. $6^{\circ}$. Si los fondos con que al pronto se cuente no bastasen, u otra circunstancia lo exigiese, podrán suprimirse de las anteriores materias las que sean menos necesarias, pero la enseñanza habrá de completarse tan luego como aquellos obstáculos desaparezcan. 
La mayor parte de las asignaturas en las EENN se referían a los saberes disciplinares, al saber a enseñar. Las de matemáticas eran «Aritmética y elementos de geometría» (en los Reglamentos de la ENC), que en el Reglamento de EENN de 1843 se concretaban un poco más: «Aritmética y sus aplicaciones, con un conocimiento general de las principales monedas, pesos y medidas que se usan en las diferentes provincias de España» $\mathrm{y}$ «Principios de geometría con sus aplicaciones a los usos comunes de la vida y de las artes industriales». El nivel de profundización en estas materias era el correspondiente a la enseñanza primaria (CARRILLO, 2005, p. 172), y los textos que se utilizaron en esta época fueron libros de texto aprobados para la enseñanza primaria (CARRILLO, 2005, p. 372-375). El R.D. de 30 de marzo de 1849, dando una nueva organización a las escuelas normales de instrucción primaria, que diferenció entre Escuelas Normales elementales (dos años de estudio) y superiores (tres años de estudio), introdujo en estas últimas una nueva materia, «Nociones de álgebra», que sobrepasaba el nivel de la enseñanza primaria e indicaba el progresivo acercamiento de los estudios de magisterio a los de la educación secundaria, más prestigiosos en esos momentos.

La materia que diferenciaba los estudios de magisterio era la Pedagogía. En los Reglamentos de la ENC se dividía en dos asignaturas: «Principios generales de educación moral, intelectual y física, con instrucciones especiales acerca de los medios más conducentes para conservar la salud de los niños y robustecerlos; o sea el modo de combinar los ejercicios gimnásticos ó corporales con juegos y ocupaciones ordinarias de la niñez», en cuyo largo título se aprecia la importancia que el médico Pablo Montesino daba a la salud de los niños y a su desarrollo corporal, y «Métodos de enseñanza y pedagogía». En el Reglamento de EENN de 1843 el título se ha resumido: «Principios de educación y métodos de enseñanza».

Los siguientes apartados están dedicados a las enseñanzas pedagógicas que impartió Montesino en la ENC, concretándolas a lo relacionado con las matemáticas.

\section{EL CURSO DE EDUCACIÓN. MÉTODOS DE ENSEÑANZA Y PEDAGOGÍA}

Las ideas de Pablo Montesino sobre la enseñanza de la aritmética fueron recogidas en su Manual para los maestros de las escuelas de párvulos (MONTESINO, 1850) y en varios artículos publicados en el BOIP, entre los que hay que destacar tres artículos dedicados a la aritmética (MONTESINO, 1843) dentro de una serie sobre métodos de enseñanza. Pero también se dispone de un valioso documento, las notas de clase que Montesino preparó para las 
asignaturas que impartía en la ENC. Estas notas de clase han sido estudiadas y publicadas bajo la dirección de Anastasio Martínez Navarro, el cual afirma sobre ellas que:

Se trata pues, y estimamos que en ello radica una gran parte de su importancia, del primer programa de pedagogía que fue explicado en la Escuela Normal Central del Reino y modelo, por tanto, dado el carácter que ésta tenía, para las demás escuelas que inmediatamente surgieron en las provincias, cuyos profesores se formaron oyendo las explicaciones de D. Pablo en Madrid, y casi sin ninguna otra fuente a la que acudir al margen de esas explicaciones (MARTÍNEZ, 1988, p. 9. El enfatizado es nuestro).

Montesino tenía el proyecto de publicar un manual a partir de estas notas, y en ellas se advierte el estado en que se encontraban los trabajos preparatorios del mismo. Martínez Navarro describe así la situación de la parte dedicada a los sistemas y métodos de enseñanza:

[...] aparece más o menos redactada; a veces tan solo esbozada de forma somera, a modo de esquemas de ideas apuntadas y no desarrolladas; frases comenzadas y seguidas, sin concluir, de puntos suspensivos; palabras clave... Casi estaríamos tentados de afirmar que se trata de auténticos guiones de notas de clase, en las que, en ocasiones, se expresan las fuentes y en cuyos márgenes figuran ideas y descriptores que facilitan la lectura y permiten seguir el hilo del discurso con una simple ojeada (MARTÍNEZ, 1988, p. 56).

En esta parte se encuentran consideraciones sobre las materias de la enseñanza primaria elemental (lectura, escritura, aritmética, gramática) y sobre geografía. A pesar de que las Escuelas Normales tenían la finalidad de formar maestros para las escuelas primarias superiores, faltan indicaciones sobre el resto de las materias de la primaria superior, entre ellas, la geometría.

La metodología de la aritmética aparece en dos contextos diferentes. En primer lugar, al tratar sobre los sistemas de enseñanza mutuo y simultáneo se describe cómo se organiza en ellos la enseñanza de la aritmética. Más adelante, al abordar las metodologías específicas de las materias de la instrucción primaria, se dedica un apartado a la aritmética.

\section{LA ENSEÑANZA DE LA ARITMÉTICA EN LOS SISTEMAS MUTUO Y SIMULTÁNEO}

En los primeros decenios del siglo XIX, la renovación de las escuelas de primeras letras se ligaba a la aplicación de un método perfecto que fuera el agente de los aprendizajes del alumnado, con independencia de la formación del maestro que estuviera a cargo de la escuela. Montesino, sin embargo, afirmaba en la introducción al Reglamento de escuelas de 1838: «sabido es que la habilidad del maestro es el gran resorte de un método, cualesquiera que sea; $\underline{\text { y que no hay buen método para un mal maestro» (Colección, 1856, p. 20). Según este }}$ 
Reglamento, el maestro debía elegir los métodos de enseñanza que considerera más adecuados a su escuela. Para ello, las EENN debían proporcionar una formación metodológica suficiente, y esto justifica el enfoque que Montesino daba a su asignatura sobre métodos de enseñanza, enfoque que ha sido calificado en ocasiones de ecléctico, pues Montesino hizo un esfuerzo de recopilación y valoración de las ideas de diferentes autores para proporcionar a los maestros unas referencias amplias que les permitieran, como se indica en el Reglamento de escuelas, elegir el método «que les parezca mas útil en sus circunstancias y mas conforme á su inclinacion» (Colección, 1856, p. 20), como guía de su actuación en el aula.

La descripción que hace Montesino de la enseñanza de la aritmética en el sistema mutuo es bastante sucinta (MONTESINO, 1988, p. 189-191). Sobre la enseñanza mutua las obras más representativas que se publicaron en España fueron la traducción del libro de Lancaster (1818), que dió a conocer el método en España, y otra que recogía la organización de la escuela de enseñanza mutua que se había creado en Madrid en 1818 (ANÓNIMO, 1820). Montesino comenta que, para la enseñanza de la aritmética, el alumnado se divide en diez secciones (como en la escuela de Madrid), pero no indica el contenido de las mismas. Presta más atención a los distintos «métodos» que se podían usar para plantear los ejercicios aritméticos. Comenta tres «métodos» que «se practican en cada sección, desde la primera hasta la última»:

1.: «mostrar la manera de hacer la cuenta», es decir, dictado por el monitor de la cuenta y su resolución;

2.: «se les exige que practiquen por sí lo que se les explicó en el primero», resolviendo cada uno de los niños la operación propuesta;

3.: «al que la saque primero»: los niños deben copiar la operación y resolverla con la mayor rapidez posible.

En la décima sección (en la que en la escuela de Madrid se estudiaban «Las demás reglas, como son los quebrados, reglas de proporción \&c.») estos ejercicios debían completarse con preguntas que les obligaran a dar razón de lo que habían hecho: «¿Qué han hecho ustedes? ¿Cómo? ¿Por qué? ¿De otro modo?»(MONTESINO, 1988, p. 190). No hemos encontrado actividades de este tipo en la propuesta de Lancaster; se trata de una aportación de Montesino que palía lo mecánico del aprendizaje de la aritmética en el sistema mutuo. Montesino apoya este proceder con varias razones:

Este examen produce un ejercicio muy útil, porque les obliga a dar la razón que han tenido para hacerlo... sino se les pone en el caso de pensar en lo que han hecho y dar razón, no aprenden mas que rutinas... por esta razón perjudiciales las reglas impresas con ejemplos al canto. Sugestiones en vez de reglas para que las saquen o deduzcan 
por inducción. No solo a fuerzas de preguntas y rodeos se les hace venir en conocimiento de ellas... Asi viene a ser un ejercicio mental que aumenta o fortifica las facultades intelectuales. (MONTESINO, 1988, p. 190).

La formulación de estas cuestiones y la valoración de las respuestas se encomienda a los Instructores; sin embargo, no se trata de una actividad en la que las respuestas del alumnado estén predeterminadas y difícilmente un alumno instructor podría dar «sugestiones en vez de reglas para que las saquen o deduzcan por inducción». En la práctica, según diversos testimonios, esta décima sección estaba formada por muy pocos alumnos o no llegaba a constituirse.

Montesino destaca la importancia de la «aritmética mental ó de memoria». Estas actividades debían plantearse sin dar al niño previamente «reglas generales y fijas»: «cada niño toma el medio que encuentra más fácil y las observaciones del maestro son las que le van llevando al más breve, que es el gran objeto» (MONTESINO, 1988, p. 190). Los primeros ejercicios se relacionan con el conocimiento de las tablas tanto de las operaciones aritméticas como de las unidades de pesos y medidas:

Comienza el instructor, por ejemplo: «6 y 8 ? 6 veces $8 »$, ¿cuántas veces 8 hay en 48 ? ¿Cuál es la $8 .^{\text {a }}$ parte de 8 ? ¿Cuántos son $2 / 8$ de 48 ? ¿Cuántos reales en tantos cuartos? ¿Cuántas pesetas? ¿Cuántos en tantos maravedises? (MONTESINO, 1988, p. 191).

Pero la finalidad era la resolución de problemas. El ejemplo propuesto es: «¿Cuánto gastaré yo en un año de 365 dias gastando 6 cuartos cada día?»; y se sugieren diferentes métodos de resolución:

Unos niños resolverán la cuestion reduciendo los 365 a cuartos y los multiplicarán por seis. Otros expertos en la tabla multiplicarán los 365 por $6=2190=257+51 / 2$ cuartos. Otro podría poner desde luego $182 \frac{1 / 2}{2}$ reales y hacer la cuenta por dos cuartos... (MONTESINO, 1988, p. 191)

Como vemos, la realización de estas actividades no sigue el modelo típico de la enseñanza mutua porque todas las estrategias de cálculo mental no pueden ser previstas de antemano. Por tanto, aunque Montesino las sitúa en los semicírculos, alude al maestro como la persona que debía dirigirlas.

A lo largo de la exposición que realiza Montesino en su Curso de Educación sobre el sistema de enseñanza simultáneo (MONTESINO, 1988, p. 192-193), son frecuentes las notas al margen que relacionan la organización de este sistema con lo previsto en el Reglamento de escuelas de 1838, que era el vigente en esos momentos; el sistema simultáneo era el más ajustado al Reglamento y el que se consideraba preferible, siempre que el número de alumnos no lo hiciera inviable, porque era el maestro el encargado directo de impartir las enseñanzas. El 
número de secciones tenía que ser menor que en la enseñanza mutua. Las que propone Montesino son las siguientes:

$1 .^{\text {a: }}$ : Aritmética mental, tablas, hacer los números.

$2 .^{\text {a: }}$ : Aritmética mental y tablas, numeración o colocación de números.

3. ${ }^{\text {a: }}$ Suma.

4. ${ }^{\mathrm{a}}$ : Resta.

5. ${ }^{\text {a: }}$ : Multiplicación.

6. ${ }^{\text {a: }}$ División.

De esta última 7. ${ }^{\mathrm{a}}$, una clase que pase a los denominados o compuestos y comience los quebrados. (MONTESINO, 1988, p. 192).

El Reglamento de escuelas de 1838 distribuía a los niños de las escuelas elementales en tres divisiones. Los contenidos de aritmética que correspondían a cada una de ellas eran los siguientes (MINISTERIO, 1979, p. 184-185):

Primera división: «contar de palabra y conocer los guarismos».

Segunda división: «hasta saber bien las cuatro primeras reglas elementales».

Tercera división: «adquirirán la práctica posible en las cuatro primeras operaciones aritméticas simples y compuestas, o en contar por números abstractos y denominados por medio de repetidas aplicaciones a los usos comunes y aprendiendo las tablas de pesos y medidas del Reino».

Si comparamos estos contenidos con los de las secciones del sistema simultáneo vemos que existe una correspondencia poco equilibrada, pues los contenidos de la primera sección y la primera división son prácticamente los mismos; a la segunda división le corresponden las secciones segunda a sexta, mientras que la hipotética séptima sección recoge los contenidos de la tercera división, tal como lo indica el propio Montesino en una nota al margen. La mayor atención a la segunda división parece relacionarse con las disposiciones del Reglamento:

Art. $57^{\circ}$. Como la clase pobre se ve frecuentemente obligada a sacar a sus hijos de la escuela demasiado pronto, procurarán los maestros promover especialmente los adelantamientos de esta segunda división, a fin de que los niños de diez años, precisados a dejar la escuela, puedan aumentar por sí, o conservar al menos con pequeño esfuerzo lo que hubieren aprendido (MINISTERIO, 1979, p. 184).

Pero constituye también un índice de la organización real de estas enseñanzas. Por ejemplo, el alumnado de la primera división (de seis a ocho años) trabajaba muy pocos conceptos numéricos durante ese periodo; seguramente se debía a la tardía incorporación a la escuela, pero también a que el acento se ponía en otros aprendizajes como la lectura, en la que se pretendía que consiguieran «leer de corrido». El agrupar en una única sección optativa, la séptima, los aprendizajes correspondientes a la tercera división, es decir, los sistemas de medida y sus operaciones, indica bien la escasez de alumnado de más de diez años, bien que los niños 
que a esa edad estuvieran escolarizados no habían alcanzado los niveles previstos en el Reglamento. Y lo más sorprendente es que esta situación fuera planteada en una asignatura impartida a futuros formadores de maestros, en la ENC, entorno en el que cabría esperar una descripción de los sistemas de enseñanza más idealizada.

La descripción que hace Montesino de la metodología de la aritmética en este sistema es bastante sucinta. No se detiene en el método a emplear, sino que remite al «indicado en el Reglamento», y de acuerdo con él indica que algunas sesiones de aritmética pueden dedicarse al cálculo mental. Para el comienzo de las sesiones de aritmética propone «tablas cantadas o recitadas, todas las secciones juntas, o cada una separadamente con instructor y maestro inspector» (MONTESINO, 1988, p. 193).

\section{ENTRE PESTALOZZI Y LA ENSEÑANZA MUTUA ${ }^{2}$}

La última parte del Curso de Educación se dedica a la metodología de las materias de la instrucción primaria, entre ellas, la aritmética (MONTESINO, 1988, pp. 212-218). En estas páginas encontramos una referencia crítica a la «manera ordinaria» de enseñar la aritmética y las ideas de Montesino sobre esta enseñanza, que revelan la influencia tanto de Pestalozzi como de la enseñanza mutua.

Supone Montesino que los alumnos de la asignatura sabían aritmética porque la habían aprendido en la clase correspondiente y que observando al profesor que la impartía, debían tener ciertas ideas sobre los métodos que podían usar. Lo que pretende Montesino en esos momentos es «explicar los diferentes medios de que se ha hecho uso para esta enseñanza elemental».

Para ello comienza con la crítica de lo que se consideraba el «método ordinario» que consistía «en aprender de memoria las tablas de sumar y multiplicar... y sobre todo y peor aprendiendo reglas. Comenzar sumando, restando, etc. números o cantidades abstractas...». Una enseñanza de este tipo se basa en la suposición «de que el adquirir estas primeras nociones es una cosa muy fácil»; Montesino califica de equivocación general esta opinión y hace observar que no todas las personas adquieren estas nociones.

Comenta la enseñanza del conteo, que se realizaba sin ninguna referencia a colecciones, dejando vacíos de significado los numerales: «se le comienza enseñando, una dos, tres, en

\footnotetext{
${ }^{2}$ Para un análisis de las propuestas del método de Pestalozzi y el de la enseñanza mutua sobre el aprendizaje matemático, puede consultarse Carrillo (2004). 
concepto de que esto es contar, para el niño seria lo mismo, silla, mesa, banco...». También señala la confusión entre número ordinal y cardinal: «para decir el significado del $\mathrm{n}^{\mathrm{o}} 3$ se enseñaba el dedo que solía contar $3 .^{\circ} \ldots{ }^{\circ}$.

En general critica que la numeración se enseñe en abstracto, sin hacer referencia a colecciones, porque así «se les enseña solo palabras y no se les dan ideas». De esta manera, los niños «no adquieren idea del valor [de posición] de las cifras». La propuesta de Montesino es que los alumnos «adquieran nociones claras de los números hasta 100 por medio de objetos» y que hagan «ejercicios analíticos» sobre números mayores, destacando el valor posicional de cada una de sus cifras y asociándolo al nombre del número. Además, «este metodo de analizar debe extenderse a la adición, sustracciones, etc.». Para que el profesor pudiera efectuar este análisis o para que los niños lo hicieran a la vista de sus compañeros, Montesino señala la importancia de usar el encerado, material escolar cuyo uso quería introducir el Reglamento de 1838 y que resulta especialmente interesante cuando el aula está organizada de acuerdo con el sistema simultáneo.

Montesino cita los algoritmos de la suma y la resta, señalando la necesidad y posibilidad de que los alumnos comprendan su justificación. Comenta la dificultad del algoritmo de la resta para los principiantes y apunta que los alumnos «lo entienden bien» cuando están «prácticos en lo anterior», es decir, cuando previamente se han realizado los ejercicios sobre numeración y se ha entendido el algoritmo de la suma.

En la organización de la enseñanza de la aritmética, Montesino reconoce lo mucho que se debe a Pestalozzi, porque «ha dado [los] principios mas sólidos para esta y otras enseñanzas, y cuyo sistema de numeración y cálculo se respeta por todos». El «gran mérito» del sistema de Pestalozzi es el ejercicio mental que requiere «a proposito para dar fuerza, perspicacia, exactitud a la razón o al razocinio», y a ello contribuye el retraso en el uso de las cifras y, por tanto, de toda la aritmética escrita «hasta que se conozcan bien los números (equivalente á cantidades)». Se destacan las ideas que Pestalozzi, en su obra Cómo Gertrudis enseña a sus hijos, había considerado básicas para plantear el aprendizaje de la aritmética:

La ciencia de la aritmetica se funda enteramente en la simple reunion y separacion de unidades.

«Una y una son dos». «Separando ó quitando una de dos queda una».

Toda operacion numerica cualquiera que sea, viene á ser una abreviacion de esta operacion elemental (MONTESINO, 1988, p. 214).

Los primeros conocimientos numéricos se deben dar a los niños «mostrándoles las variaciones de más o menos por medio de objetos reales presentes a la vista»; de esta forma se 
obtiene sobre los números un «conocimiento claro, intuitivo de sus reales propiedades y proporciones para fundar sobre él todo el progreso posterior». Pestalozzi previene contra el uso prematuro de los símbolos aritméticos, que él llamaba «abreviaciones aritméticas», porque consideraba que obscurecían y confundían la intuición sobre el número.

El uso de colecciones se considera particularmente importante para que los niños adquieran las ideas sobre los diez primeros números. Pestalozzi «se valió de diferentes objetos para ejercitarlos en el número 1 , en el 2 , etc. de mil maneras» hasta conseguir que los niños las percibieran «distinta y claramente sin necesidad de raciocinio». En este punto, Montesino remite al «método adoptado en las escuelas de párvulos», reconociendo así su deuda como autor del Manual para los maestros de las escuelas de párvulos con respecto a Pestalozzi. También se citan y valoran positivamente, aunque no se mencione ningún ejercicio sobre ellas, las tres tablas de Pestalozzi para el aprendizaje de la aritmética.

En Pestalozzi encuentra Montesino la fundamentación adecuada para la metodología de la aritmética y los ejercicios que pueden plantearse para iniciar su aprendizaje. En el sistema pestalozziano lo fundamental es que los niños adquieran la intuición del número y de sus descomposiciones, y para ello se potencia la aritmética mental. También se habla del interés de la aritmética escrita, pero frente a la prolijidad con que se aborda la sucesión de ejercicios de aritmética mental, se suele señalar simplemente que, después de haber adquirido las ideas sobre el número, los discípulos pueden abordar la aritmética escrita.

Tal vez por ello, cuando Montesino plantea la enseñanza de la aritmética en las escuelas y la organización del aprendizaje de los algoritmos recomienda el método «adoptado en la Enseñanza mutua»y remite a lo que ha dicho anteriormente sobre la aritmética en ese sistema, pero tanto aquí como cuando lo describía, Montesino introduce actividades diferentes a las que se encontraban en los tratados clásicos sobre la enseñanza mutua; con esas actividades plantea una enseñanza de la aritmética menos mecánica y fomenta el razonamiento de los alumnos. Como ya hemos señalado, muchas de esas actividades suponen plantear cuestiones sobre el sentido y las técnicas relativas a la numeración, los algoritmos, los procedimientos de cálculo mental o la resolución de problemas, y valorar respuestas que no pueden ser determinadas de antemano; son tareas que sobrepasan la competencia de un alumno instructor y que requieren la actuación directa del maestro. Por tanto, aunque Montesino no critica el método de enseñanza mutua sino que lo recomienda, las variaciones que introduce en él cambian sustancialmente sus presupuestos. 
Las ideas sobre el aprendizaje de la aritmética que Montesino expone sucintamente en el Curso se encuentran más desarrolladas en otros escritos como el Manual para los maestros de las escuelas de párvulos (MONTESINO, 1850), publicado en 1840 en el primer año de funcionamiento de la ENC, y en los artículos que, sobre la enseñanza de la aritmética, publicó Montesino en el BOIP (MONTESINO, 1843). Las concordancias que existen entre estos artículos y el Curso de Educación sugieren que reflejan el contenido de las clases de Montesino en la ENC. En el primero de los artículos se justifica la importancia de los conocimientos aritméticos para toda clase de personas, se critican los métodos usuales de su enseñanza, repitiendo las razones expuestas en el Curso de Educación, fundamenta la enseñanza de la aritmética en Pestalozzi, incluyendo una larga cita del mismo y recomienda el uso de los tableros contadores para conseguir una idea intuitiva de la numeración en la primera centena; también hay ejercicios sobre estos números y sobre medidas de longitud y peso. En los siguientes artículos hay propuestas sobre la enseñanza de los algoritmos de las operaciones aritméticas basadas en el análisis de los números de varias cifras, lo que permitía presentar los algoritmos de forma razonada, no como un conjunto de reglas arbitrarias. Sobre los algoritmos usuales se presentaban variaciones que constituyen procedimientos más lógicos o más detallados que aquellos, y así se considera que:

[...] una enseñanza de esta especie dirigida con discernimiento y juicio convencerá prácticamente al discípulo de que las operaciones aritméticas se fundan en principios que desenvueltos convenientemente no pueden menos de producir resultados importantes. (MONTESINO, 1843, p. 187).

\section{LOS SABERES PROFESIONALES DE LOS MAESTROS QUE ENSEÑNAN MATEMÁTICAS}

La organización propuesta por Montesino para la ENC y las EENN provinciales, así como los correspondientes Planes de estudio supusieron una ampliación de los tipos de saberes que se consideraban necesarios para la formación de los maestros. En este apartado se sintetiza su propuesta usando las categorías comentadas en la introducción del trabajo y refiriéndolas al maestro como docente de matemáticas en la enseñanza primaria.

1) la matemática $a$ enseñar, es decir, los contenidos matemáticos de la enseñanza primaria se cursaban en asignaturas específicas: «Aritmética y sus aplicaciones, con un conocimiento general de las principales monedas, pesos y medidas que se usan en las diferentes 
provincias de España»y «Principios de geometría con sus aplicaciones a los usos comunes de la vida y de las artes industriales».

2) los saberes para enseñar, las herramientas que necesita el maestro para su trabajo docente, se han clasificado en tres tipos:

2a) Matemática para enseñar, que requiere una profundización de la matemática $a$ enseñar, es abordada por Montesino en los artículos publicados en el BOIP (MONTESINO, 1843), pero limitándose a la numeración y las operaciones aritméticas. En estos artículos, se relaciona el aprendizaje de la numeración y las operaciones aritméticas, se hacen consideraciones sobre su importancia, se presentan los algoritmos de forma justificada así como la posibilidad de técnicas alternativas.

2b) La metodología de las matemáticas se limita a algunos aspectos de una metodología de la aritmética y es tratada, fundamentalmente, en el Curso de educación, basada en las propuestas de Pestalozzi y, en parte, de la enseñanza mutua.

2c) El Curso de educación, como indica el título de las asignaturas a la que responde, también recoge saberes pedagógicos generales que no han sido objeto de este trabajo.

\section{CONSIDERACIONES FINALES}

Pablo Montesino fue un experto al que recurrió el Gobierno entre 1834 y 1849, época en la que el Estado estaba creando un sistema público de enseñanza. Como señalan Morais y Valente (2020), citando el libro de Hofstetter, La fabrique des savoirs: figures et pratiques d'experts, es en los periodos en los que el Estado se hace cargo de nuevas responsabilidades con respecto al sistema educativo cuando se recurre a expertos que asesoren y generen documentos para apoyar los cambios; es lo que ocurrió con Pablo Montesino. Los cargos que desempeñó y su influencia en la redacción de las leyes que sobre la enseñanza primaria se elaboraron en esta época, confirieron prestigio e importancia a sus ideas y, por ello, pudo actuar como introductor y como difusor de nuevas formas de enseñanza, en particular, de la aritmética, pues Montesino defiende nuevas formas de enseñar aritmética en las que se tiene en cuenta al niño y su forma de aprender.

Como director de la ENC, fue el primer profesor de Pedagogía que hubo en España y, por tanto, la primera persona que impartió contenidos sobre Didáctica de las Matemáticas. Al ser la Pedagogía un contenido nuevo en la formación de los maestros, Montesino tuvo que construirla como asignatura, y sus enseñanzas fueron el inicio de estos estudios en España; en 
particular, hay que considerar sus enseñanzas como el punto de partida de la Didáctica de las Matemáticas en las Escuelas Normales en España.

Montesino toma de Pestalozzi una teoría sobre el aprendizaje infantil que enfatiza el papel de la intuición. Como consecuencia, señala la importancia de los primeros aprendizajes aritméticos y la conveniencia de que se realicen a partir de colecciones de objetos para proporcionar a los niños una base intuitiva de los conceptos numéricos.

La iniciación en las operaciones aritméticas la une Montesino a la resolución de problemas, que son justamente los que pueden permitir al niño dar sentido a estas operaciones. Resulta también interesante su insistencia en el aprendizaje de técnicas de cálculo mental que permiten operar sin necesidad de lápiz y papel.

El aprendizaje del sistema de numeración se consideraba muy importante por ser básico para la comprensión de la aritmética. Montesino lo basaba en la comprensión de la decena y la centena por medio de ejercicios en el tablero de contar y en un «método analítico» de la representación oral y escrita de los números de varias cifras. La comprensión del sistema de numeración permitía abordar de forma razonada el aprendizaje de los algoritmos de las operaciones aritméticas; para ello, en los artículos del BOIP plantea variantes de esos algoritmos más lógicas en las que se especificaban las operaciones intermedias a realizar.

Sus ideas sobre la enseñanza de la aritmética supusieron un gran avance con respecto a las prácticas habituales en esos momentos en las escuelas, pero no forman un cuerpo coherente, pues no logró una síntesis aplicable a todos los aspectos de la enseñanza de la aritmética en la escuela primaria. No era esa su especialidad; sus preocupaciones e intereses en el campo de la educación eran más generales, y eso acrecienta el mérito de sus propuestas. No consigue integrar su propuesta sobre la iniciación de cada uno de los conocimientos aritméticos propios de la enseñanza primaria con la organización del aula necesaria para ello y para que los niños consigan el dominio de las técnicas asociadas a dichos conocimientos: no consigue integrar Pestalozzi y la enseñanza mutua.

Por ejemplo, critica el aprendizaje de las tablas de memoria y, sin embargo, las propone tanto en el Manual como en el Curso. Valora en alto grado el uso de la intuición para la iniciación en la aritmética, pero no todos sus ejercicios son intuitivos. Además, propone el uso de algoritmos de las operaciones aritméticas que puedan ser justificados a los alumnos y, más adelante, acepta la propuesta de la enseñanza mutua, que implica un aprendizaje mecánico de los algoritmos usuales. 
Si se consideran los contenidos del Curso de Educación, incluso complementados con los artículos del BOIP, se advierte la existencia de grandes lagunas que ponen en entredicho la formación sobre las matemáticas para enseñar y la metodología de las matemáticas que recibían los encargados de la formación de los maestros de toda España. Por ejemplo, no responde claramente a la cuestión de cómo iniciar el aprendizaje de la aritmética en las escuelas primarias. Remite a las ideas de Pestalozzi y al Manual de párvulos, pero no da indicaciones sobre la implementación de esas ideas en el nivel educativo primario, nivel que organizaba sus enseñanzas de una forma muy distinta a las escuelas de párvulos.

Las EENN, sobre todo en su primera época, tenían la misión de formar maestros de escuela superior y, sin embargo, no hay ninguna indicación sobre la enseñanza de los conocimientos matemáticos correspondientes a este nivel; está ausente toda referencia a las razones, proporciones y regla de tres, e incluso a contenidos de la enseñanza primaria elemental como fracciones y números decimales. También está ausente en el Curso de Educación toda indicación sobre la enseñanza de los sistemas de medida.

Las enseñanzas de Montesino en la ENC fueron un referente para el profesorado de las EENN que se formó en ese centro. La novedad de la materia, la ausencia de obras de Pedagogía en castellano y el prestigio personal de Montesino ante sus alumnos hizo que sus enseñanzas fueran prácticamente la única referencia sobre Pedagogía que tuvieron estos profesores, los cuales, en sus clases tuvieron como modelo la organización y los contenidos que Montesino había dado a la asignatura. Los libros de texto sobre Pedagogía que escribieron siguen ese modelo. Joaquín Avendaño y Mariano Carderera, dos alumnos de las primeras promociones de la ENC que llegaron a ser Inspectores Generales de primera enseñanza, escribieron un Curso elemental de Pedagogía (AVENDAÑO et al., 1850), que fue recomendado oficialmente y utilizado en muchas EENN (CARRILLO, 2005, p. 322-324). El esquema y los contenidos siguen de cerca el Curso de Educación y, en lo que se refiere a la enseñanza de la aritmética, recoge, resumido y repitiendo ejemplos, el contenido de los artículos que publicó Montesino en el BOIP (MONTESINO, 1843); como novedad, añade la descripción del tablero contador de quebrados y un párrafo sobre la enseñanza de la geometría destacando su carácter práctico.

La aceptación del modelo de enseñanzas pedagógicas por los discípulos de Pablo Montesino, profesores de las EENN, indican su creciente institucionalización, y se convirtieron en una referencia para la formación del magisterio y para la docencia en la enseñanza primaria. Concluyendo, las aportaciones de Pablo Montesino fueron el referente sobre los saberes 


\section{REAMEC

profesionales de los maestros como docentes de matemáticas en las escuelas primarias en el segundo tercio del siglo XIX en España.

\section{REFERENCIAS}

ALMEIDA, André Francisco; VALENTE, Wagner Rodrigues. Os experts e a produção de saberes para a docência: primeiros estudos do acervo Lydia Lamparelli. Linhas Críticas, Brasilia, v. 25, p. 318-332, fev./feb. 2019. https://doi.org/10.26512/lc.v25.2019.23109

ANÓNIMO. Método de enseñanza mutua, según los sistemas combinados del Dr. Bell y de Mr. Lancaster, para uso de las escuelas elementales ó de primeras letras; aprobado por S.M., y mandado observar en la escuela central de Madrid y en las demás que se esta clase se establezcan en España. Madrid: Imprenta Real, 1820.

AVENDAÑO, Joaquín; CARDERERA, Mariano. Curso elemental de Pedagogía. Madrid: Im. A. Vicente, 1850.

BALL, Deborah Loewenberg; THAMES, Mark Hoover; PHELPS, Geoffrey. Content knowledge for teaching: what makes it special? Journal of Teacher Education, Washington, n. 59, v.5, p. 389-407, nov. 2008.

BURKE, Peter. ¿Qué es la historia del conocimiento? Cómo la información dispersa se ha convertido en saber consolidado a lo largo de la historia. Buenos Aires: Siglo Veintiuno, 2017.

CARRILLO GALLEGO, Dolores. La codeterminación entre las organizaciones matemáticas y las organizaciones didácticas. Pestalozzi y la enseñanza mutua. Recherches en Didactique des Mathématiques, París, v. 24, n.1, p. 11-44, 2004. Disponible en: https://revue$\underline{\text { rdm.com/2004/la-codeterminacion-entre-las/ }}$

CARRILlO GALLEGO, Dolores. La Metodología de la aritmética en los comienzos de las Escuelas Normales (1838-1868) y sus antecedentes. Tesis Doctoral-Facultad de Educación, Universidad de Murcia, 2005.

CARRILlO GALLEGO, Dolores. Renovando las matemáticas escolares por medio de la formación del magisterio. In LEME DA SILVA, María Celia; PINTO, Thiago Pedro (org.) História da Educação Matemática e Formação de Professores: aproximaçoes possiveis. Sao Paulo: Livraria da Física, 2020. p. 53-89.

CIRADE, Gisèle. Devenir professeur de mathèmatiques: entre problèmes de la profession et formation en IUFM. Les mathématiques comme problème professionnel, Tesis Doctoral - Université Aix-Marseille I, Université de Provence, 2006.

Colección Legislativa de Instrucción Primaria. Madrid: Imprenta Nacional, 1856.

ESCOLANO BENITO, Agustín. Escuela y sociedad en la revolución liberal española. In VEGA GIL, Leoncio (ed.). Pablo Montesino y la modernización educativa en España. Zamora: Instituto de Estudios Zamoranos (CSIC). 1998. p. 15-22. 
ESCOLANO BENITO, Agustín. Las escuelas normales, siglo y medio de perspectiva histórica. Revista de Educación, Madrid, n. 269, p. 55-76, 1982.

Gaceta de Madrid, nº 910, 31 de Mayo de 1837.

GIL DE ZÁRATE, Antonio. De la Instrucción pública en España. Tomo I. Madrid: Imprenta del Colegio de Sordo-mudos, 1855.

HOFSTETTER, Rita; SCHNEUWLY, Bernard. Savoirs en (trans)formation. Au coeur des professions de l'enseignement et de la formation. Bruxelles: De boeck, 2007.

LANCASTER, Joseph Sistema Inglés de instrucción o colección completa de las invenciones y mejoras puestas en práctica en las escuelas reales de Inglaterra. Traducido del francés por Pedro Ferrer y Casaús. Madrid: Imprenta de la calle de la Greda, 1818.

MARTÍNEZ NAVARRO, Anastasio. Estudio preliminar. In MONTESINO, Pablo. Curso de Educación y Métodos de Enseñanza y Pedagogía. Madrid: MEC, 1988.

MINISTERIO DE EDUCACIÓN. Historia de la Educación en España. Tomo II. De las Cortes de Cádiz a la Revolución de 1868. Madrid: Ministerio de Educación, 1979.

MONTESINO, Pablo. Ligeros apuntes y observaciones sobre la instrucción secundaria o media y la superior o de Universidad. Madrid: Im. de Sajo, 1836.

MONTESINO, Pablo. Métodos especiales de enseñanza. Enseñanza de la aritmética. Boletín Oficial de Instrucción Pública, V, pp. 77-84, 122-128, 178-190, 1843.

MONTESINO, Pablo. Manual para los maestros de escuelas de párvulos. Madrid: Im. del Colegio de Sordo-mudos y Ciegos, 1850.

MONTESINO, Pablo. Manual para los maestros de escuelas de párvulos. Madrid: CEPE, 1992. Introducción crítica de Julio Ruiz Berrio.

MONTESINO, Pablo. Curso de Educación y Métodos de Enseñanza y Pedagogía. Madrid: MEC, 1988. Edición, estudio preliminar y notas de Anastasio Martínez Navarro.

MORAIS, Rosilda dos Santos; VALENTE, Wagner Rodrigues. Os Experts e o saber profisional do profesor que ensina matemática. Ciência \& Educação, Bauru, v. 26, 2020. https://doi.org/10.1590/1516-731320200029

Reglamento de la Escuela Normal Seminario Central de Maestros de Instrucción Primaria. Imprenta Nacional, Madrid, 1842.

RUIZ BERRIO, Julio. Introducción crítica. In MONTESINO, Pablo. Manual para los maestros de escuelas de párvulos. Madrid: CEPE, 1992. p. 9-32.

SHULMAN, L. S. Knowledge and teaching: Foundations of the new reform. Harvard Educational Review, n. 57, 1987. p. 1-22. 
VALENTE, Wagner Rodrigues. El saber profesional del profesor que enseña matemática: el futuro del pasado. Paradigma, v. 39, n. extra 1, 2018. p. 190-201. Disponible en: http://revistaparadigma.online/ojs/index.php/paradigma/article/view/674

VEGA GIL, Leoncio (ed.). Pablo Montesino y la modernización educativa en España. Zamora: Instituto de Estudios Zamoranos (CSIC). 1998.

\section{APÉNDICE}

\section{AGRADECIMIENTOS}

No aplica.

\section{FINANCIACIÓN}

No aplica.

CONTRIBUCIONES DE LA AUTORIDAD

Ambos autores: Dolores Carrillo Gallego y José Ginés Espín Buendía.

\section{CONFLICTOS DE INTERÉS}

Los autores han declarado que no existe ningún conflicto de intereses de carácter personal, comercial, académico, político y económico con respecto a este manuscrito.

\section{DISPONIBILIDAD DE DATOS DE BÚSQUEDA}

Los autores pondrán a disposición los datos de la investigación si les son solicitados.

CONSENTIMIENTO PARA USAR IMAGEN

No aplica

\section{APROBACIÓN DEL COMITÉ DE ÉTICA DE INVESTIGACIÓN \\ No aplica}

\section{CÓMO CITAR - ABNT}

CARRILlO GALLEGO, Dolores; ESPÍN BUENDÍA, José Ginés. Pablo Montesino y la formación matemática del magisterio en España (1838-1850). REAMEC - Rede Amazônica de Educação em Ciências e Matemática. Cuiabá, v. 9, n. 3, e21087, set./dez, 2021. https://doi.org/10.26571/reamec.v9i3.13012

\section{CÓMO CITAR - APA}

Carrillo Gallego, D. \& Espín Buendía, J.G. (2021). Pablo Montesino y la formación matemática del magisterio en España (1838-1850). REAMEC - Rede Amazônica de Educação em Ciências e Matemática, 9(3), e21087. https://doi.org/10.26571/reamec.v9i3.13012

\section{LICENCIA DE USO}

Con licencia de Creative Commons Attribution-NonCommercial 4.0 International License (CC BY-NC 4.0). Esta licencia le permite compartir, copiar, redistribuir el manuscrito en cualquier medio o formato. Además, permite adaptar, remezclar, transformar y construir sobre el material, siempre que se dé el debido crédito por la autoría y publicación inicial en esta revista.

\section{DERECHOS DE AUTOR}

Los derechos de autor son mantenidos por los autores, quienes otorgan a REAMEC - Red Amazónica para la Educación Científica y Matemática - los derechos exclusivos de primera publicación. Los autores no serán remunerados por la publicación de trabajos en esta revista. Los autores están autorizados a asumir contratos adicionales por separado, para la distribución no exclusiva de la versión del trabajo publicado en esta revista (ej .: publicación en un repositorio institucional, en un sitio web personal, publicación de una traducción, o como capítulo de un libro), con reconocimiento de autoría y publicación inicial en esta revista. Los editores de la Revista tienen derecho a realizar ajustes textuales y adaptarlos a las reglas de la publicación. 


\section{PUBLISHER}

Universidad Federal de Mato Grosso. Programa de Posgrado en Educación en Ciencias y Matemáticas (PPGECEM) de la Red Amazónica de Educación en Ciencias y Matemáticas (REAMEC). Publicación en el portal de revistas de la UFMT. Las ideas expresadas en este artículo son responsabilidad de sus autores y no necesariamente representan la opinión de los editores o de la universidad.

\section{EDITOR RESPONSABLE}

\section{Dailson Evangelista Costa (D) 9}

\section{HISTÓRICO}

Enviado: 21 de septiembre de 2021.

Aprobado: 15 de octubre de 2021.

Publicado: 06 de noviembre de 2021. 